Revue

Revue de l'histoire des religions

del'histoire des religions

2| 2016

La représentation juive de l'empire romain comme pendant et frère jumeau d'Israël. Histoire et enjeux

The Rabbis Write Back! L'enjeu de la "parenté » entre Israël et Rome-Ésaü-Édom

The Rabbis Write Back! The stakes of the "kinship" between Israel and Rome-

Esau - Edom

Katell Berthelot

\title{
OpenEdition
}

Journals

Édition électronique

URL : http://journals.openedition.org/rhr/8552

DOI : 10.4000/rhr.8552

ISSN : 2105-2573

Éditeur

Armand Colin

Édition imprimée

Date de publication : 1 juin 2016

Pagination : 165-192

ISBN : 978-2-200-93060-8

ISSN : 0035-1423

Référence électronique

Katell Berthelot, «The Rabbis Write Back! L'enjeu de la « parenté » entre Israël et Rome-Ésaü-Édom », Revue de l'histoire des religions [En ligne], 2 | 2016, mis en ligne le 01 juin 2019, consulté le 05

septembre 2020. URL : http://journals.openedition.org/rhr/8552 ; DOI : https://doi.org/10.4000/rhr 8552 


\section{The Rabbis Write Back! L'enjeu de la « parenté » entre Israël et Rome-Ésaü-Édom}

Au vu des parentés mythiques attestées dans la Bible d'une part, et dans le monde hellénistique de l'autre, l'identification de Rome avec Ésaü ou Édom ne reflète pas l'invention d'une parenté, mais plutôt la création d'une typologie, pour le moins paradoxale puisqu'elle implique une proximité et un lien intime entre Israël et Rome. Pour certains historiens, cette typologie ne ferait sens que dans le contexte de la christianisation de l'empire. L'examen des sources montre cependant que l'idée de la gémellité et de la rivalité entre Israël et l'empire romain tire plutôt son origine des défaites juives contre Rome et de la perception, par les rabbins, d'une prétention de Rome à usurper la place concrète et symbolique d'Israël dans le monde.

\section{The Rabbis Write Back! The stakes of the "kinship" between Israel and Rome - Esau - Edom}

Compared with the mythical kinships found in the Bible as well as in the Hellenistic world, the identification of Rome with Esau or Edom does not reflect the invention of a kinship between Romans and Jews, but rather the creation of a typology. The latter is all the more paradoxical as it implies a proximity and even an intimacy between Israel and Rome. According to some historians, this typology makes sense only with the christianization of the empire. However, a close examination of the sources shows that the idea of Israel and Rome as twins and rivals originates in the Jewish defeats against Rome and in the perception by the rabbis of Rome as usurping the concrete and symbolic place of Israel in the world.

* TDMAM (UMR 7297), Maison Méditerranéenne des Sciences de l'Homme, 13090 Aix-en-Provence, France. Cette recherche a été financée par le Conseil Européen de la Recherche (European Research Council) dans le cadre du $7^{\text {e }}$ programme-cadre de l'Union européenne (FP/2007-2013) et du programme ERC Judaism and Rome (ERC Grant Agreement n. 614 424). 


\section{INTRODUCTION}

L'idée d'une parenté entre Israël et Rome, exprimée à travers l'identification de Rome avec Ésaü, le frère jumeau d'Israël - Jacob, lui-même identifié à Édom, peuple voisin d'Israël à l'époque biblique, a suscité bon nombre d'interrogations et de débats, qui sont loin d'être clos. Plusieurs théories ont été élaborées pour expliquer ce curieux rapprochement.

Une première hypothèse, avancée entre autres par Louis Ginzberg, fait remonter l'origine de la parenté entre Israël et Rome à la période du roi Hérode, dont les ancêtres étaient iduméens, c'està-dire édomites, et qui fut un roi client de Rome' ${ }^{1}$. Ce serait par le biais de la figure d'Hérode qu'un lien aurait été établi entre Édom (ou l'Idumée) et Rome. Aujourd'hui cette hypothèse est cependant écartée par la plupart des chercheurs, à l'exception notable d'Irit Aminoff, qui, dans sa thèse de doctorat, intitulée The Figures of Esau and the Kingdom of Edom in Palestinian Midrashic-Talmudic Literature in the Tannaic and Amoraic Period, accorde quelque crédit à cette piste hérodienne, tout en reconnaissant que ce n'est pas totalement démontrable ${ }^{2}$.

Dans un article fondamental publié en 1969, Gerson Cohen a suggéré que les passages concernant Édom dans les Lamentations de Jérémie (en lien avec la destruction du Premier Temple) correspondaient si parfaitement à la représentation que se faisaient

1. Voir Louis Ginzberg, The Legends of the Jews, Philadelphia, Jewish Publication Society, 1968, vol. V, p. 272, note 19: « The use of the name Edom, Seir, Esau, and similar ones, to describe Rome is very old, and was probably coined at the time of Herod, whose designation "the Idumean" was applied to his masters, the Romans ».

2. Irit Aminoff, The Figures of Esau and the Kingdom of Edom in Palestinian Midrashic-Talmudic Literature in the Tannaic and Amoraic Period, $\mathrm{PhD}$, Melbourne University, 1981, p. 225-233. Voir déjà Rubens Duval, « Notes sur la Peschitto I. Édom et Rome», Revue des Etudes Juives 14, 1887, p. 49-51. Cette théorie est également adoptée (en passant) par André Lemaire, «D’Édom à l'Idumée et à Rome », Des Sumériens aux Romains d'Orient : la perception géographique $d u$ monde, dir. Arnaud Sérandour, Paris, Jean Maisonneuve - Librairie d'Amérique et d'Orient, 1997, p. 81-103 (voir p. 100). L'explication faisant intervenir Hérode est déjà rejetée par Moshe David Herr dans sa thèse de doctorat, Le pouvoir romain dans la littérature des Tannaïm, Université hébraïque de Jérusalem, 1970, p. 116 et 123 (en hébreu). 
les Juifs des Romains après la destruction du Second Temple, que le parallèle entre les deux s'imposait ${ }^{3}$. Cohen voit en particulier dans Lamentations 4, 21-22 un message de consolation que les Juifs de la fin du $\mathrm{I}^{\mathrm{er}}$ siècle de $\mathrm{n}$. è. et du début du $\mathrm{II}^{\mathrm{e}}$ siècle interprétèrent en lien avec Rome ${ }^{4}$. Il en découle une autre explication possible du lien établi entre Édom et Rome : l'existence de nombreux oracles de jugement et de destruction contre Édom au sein du corpus biblique. Le cas le plus exemplaire est celui du Livre d'Abdias, le plus court de la Bible hébraïque (21 versets seulement), qui est entièrement tourné contre Édom. Les versets 1 à 9 annoncent le châtiment d'Édom - Ésaü, les versets 10 à 16 expliquent ce châtiment par l'attitude des Édomites vis-à-vis d'Israël, explicitement présenté comme leur frère ; enfin, la dernière partie (vv. 17-21) annonce le rétablissement d'Israël et la conquête des territoires voisins. Cette explication de l'identification de Rome avec Ésaü, en lien avec les oracles de jugement contre Édom, a été proposée par de nombreux chercheurs : non seulement Gerson Cohen, mais aussi Moshe David Herr, Irit Aminoff, Mireille Hadas-Lebel et d'autres ${ }^{5}$.

On pourrait aussi envisager que la proximité phonétique des deux noms, «Édom » et « Rome », ait joué un rôle dans le rapprochement effectué par les rabbins. De tels jeux phonétiques, peu soucieux d'exactitude étymologique, sont attestés dans la littérature rabbinique. On rencontre ainsi un jeu de mots entre Doumah (nom qui apparaît en Isaïe 21, 11 en lien avec Séir, le territoire d'ÉdomÉsaui) et Roma/Romi, où la différence joue surtout sur une lettre (רומה et / רומה) ; dans le Talmud de Jérusalem, il est question d'un livre de Rabbi Meir où celui-ci aurait écrit: «oracle sur Doumah, oracle sur Rome » (y. Ta'anit 1.1 [64a], d'après le ms Leiden Scaliger 3). Jérôme connaissait ce jeu de mots rabbinique

3. Gerson Cohen, «Esau as Symbol in Early Medieval Thought», Jewish Medieval and Renaissance Studies, dir. Alexander Altmann, Cambridge, Harvard University Press, 1967, p. 19-48 (voir p. 24-25).

4. Lm 4, 21-22 : «Égaie-toi, réjouis-toi, Édom la belle, habitante du pays de 'Utz ! À toi aussi on passera la coupe, tu t'enivreras et tu te mettras à nu. Ta faute est à son terme, Sion la belle ; il ne t'exilera plus. Il te fera rendre des comptes pour ta faute, Édom la belle! Il mettra tes péchés à découvert ». Les traductions des textes bibliques sont inspirées de la traduction de la Nouvelle Bible Segond, tout en en divergeant si nécessaire.

5. Voir G. Cohen, « Esau as Symbol », p. 24-25. 
et y fait allusion dans ses commentaires sur Abdias et Isaïe ${ }^{6}$. Un texte tardif attribue aussi à ce même Rabbi Meir une lecture d'Isaïe 34, 7 (« Les buffles tombent avec eux ») dans laquelle il substitue 《Romains » (romyim [רומיים]) à «buffles» (reémim [ראמים]) (Pesikta deRav Kahana, Vayehi Bahatzi 11) ${ }^{7}$.

Une autre théorie met en avant le rôle joué par le christianisme. Dans son livre Persia and Rome in Classical Judaism ${ }^{8}$, Jacob Neusner avance l'idée qu'avant le $\mathrm{IV}^{\mathrm{e}}$ siècle et la christianisation de l'empire, Rome ne représentait qu'un lieu ou un empire parmi d'autres pour les rabbins, et qu'elle ne revêtait pas d'importance particulière : «To invoke a modern category, Rome stood for a perfectly secular matter: a place, where things happened. Rome in no way symbolized anything beyond itself $»^{9}$. À partir du IV siècle, la christianisation de l'empire aurait changé la donne : "Christian Rome posed a threat without precedent $»^{10}$. Dans le grand midrash sur la Genèse, Bereshit (Genèse) Rabbah, daté d'environ 400, les très nombreuses évocations de Rome en lien avec la figure d'Ésaü - Édom seraient donc intimement liées à la polémique contre le christianisme. Ce serait en définitive le défi nouveau posé par le christianisme au judaïsme qui aurait poussé les rabbins à identifier Rome au frère jumeau d'Israël.

Afin d'y voir plus clair dans cet éventail de propositions, je me propose de re-situer tout d'abord la parenté fictive entre Israël et Rome dans la longue durée, c'est-à-dire dans la perspective des parentés bibliques et des syngeneiai de l'époque hellénistique, afin de mieux cerner la nature de l'identification de Rome avec Ésaü - Édom, et sa nouveauté. J'aborderai ensuite la question de l'origine de cette identification et du ou des sens que l'on peut lui attribuer.

6. Voir l'article d'Hervé Inglebert dans ce dossier.

7. Ms Oxford, Bodleian Library, 2339. Cf. Friedrich Avemarie, « Esaus Hände, Jakobs Stimme : Edom als Sinnbild Roms in der frühen rabbinischen Literatur », Die Heiden, dir. Reinhard Feldmeier, Tübingen, Mohr Siebeck, 1994, p. 177-208 (voir p. 182).

8. Publié originellement en 1984. J'utilise la réédition de 2008 (Lanham, University Press of America). Neusner a également exprimé cette position dans le volume Judaism in the Matrix of Christianity (première édition Philadelphia, Fortress Press, 1986 ; réédition Atlanta, Scholars Press, 1991) ; voir en particulier le chapitre 5, "Change in the Symbolization of the Other: Rome from Place to Sibling », p. 73 à 87 de l'édition de 1991.

9. J. Neusner, Judaism in the Matrix of Christianity, p. 77.

10. J. Neusner, ibid., p. 78. 


\section{LeS PARENTÉS MYTHIQues dANS LA BiBLE}

Les parentés mythiques abondent dans la Bible hébraïque. Elles concernent le plus souvent les voisins immédiats d'Israël, comme les Ammonites et les Moabites, situés sur la rive orientale du Jourdain, qui sont décrits comme de lointains descendants de Lot, le neveu d'Abraham (Gn 19, 36-38). Les Édomites, autre peuple installé à l'origine en Transjordanie, autour du Mont Séir, sont pour leur part considérés comme des descendants d'Ésaü, tout comme les Amalécites. Dès le Livre de la Genèse, Ésaü luimême est identifié avec Édom. Le verset Genèse 25, 30 propose une étymologie fantaisiste du nom « Ésaü » en lien avec la couleur rouge, adom en hébreu : «Ésaü dit à Jacob: Laisse-moi, je te prie, manger de (ce potage) rouge (ha-adom) - oui, ce (potage) rouge (ha-adom ha-zèh) - car je suis épuisé. C'est pourquoi on l'a appelé du nom d'Édom (Rouge) ». Ésaü accepte de céder son droit d'aînesse à son frère cadet en échange de «ce (potage) rouge » (Gn 25, 31-35). Ésaü est en outre décrit comme résidant au pays d'Édom : «Jacob envoya, en avant de lui, des messagers à Ésaü, son frère, à Séir, au pays d'Édom » (Gn 32, 4). Par la suite, lorsque le texte énumère les descendants d'Ésaü, il identifie explicitement Ésaü et Édom : «Voici la généalogie (les engendrements) d'Ésaü, qui est Édom » (Gn 36, 1). En Genèse 36, 8, nous lisons à nouveau : «Ésaü s'installa dans la région montagneuse de Séir ; Ésaü, c'est Édom »; et en Gn 36, 9 : «Voici la généalogie (les engendrements) d'Ésaü, père d'Édom, dans la région montagneuse de Séir ».

Cette identification d'Ésaü avec Édom et des Édomites avec les descendants d'Ésaü figure dans plusieurs livres bibliques, certains passages insistant sur le lien de fraternité qui en découle pour les Édomites et les Israélites ${ }^{11}$. Dans le Livre des Nombres, lorsque

11. Pour une présentation détaillée des traditions bibliques relatives à Ésaü-Édom et à son lien de fraternité avec Israël, voir John R. Bartlett, «The Brotherhood of Edom », Journal for the Study of the Old Testament 4, 1977, p. 2-27; Diana Vikander Edelman, You shall not Abhor and Edomite for he is your Brother: Edom and Seir in History and Tradition, Atlanta, Scholars Press (Archaeology and Biblical Studies Series, 3), 1995 ; Elie Assis, « Why Edom ? On the Hostility towards Jacob's Brother in Prophetic Sources », Vetus Testamentum 56/1, 2006, p. 1-20; et la synthèse récente de Bradford A. Anderson, Brotherhood and Inheritance: A Canonical Reading of the Esau and Edom Traditions, New York - London, T\&T Clark, 2011. 
Moïse et les enfants d'Israël s'apprêtent à traverser le territoire des Édomites, Moïse envoie depuis Qadesh des messagers au roi d'Édom pour lui dire : «Ainsi parle Israël, ton frère (ahikha Israel) : Tu sais toutes les choses pénibles qui nous ont atteints $[\ldots] »(\mathrm{Nb} 20,14)$. Bien que Moïse soit prêt à payer l'eau bue sur le territoire d'Édom par les Israélites et leurs troupeaux, le roi d'Édom refuse de les laisser passer et vient à leur rencontre avec une armée, de telle sorte qu'Israël doit se résigner à emprunter un autre chemin $(\mathrm{Nb} 20,21)$. Malgré ce manquement grave aux devoirs de l'hospitalité, le Livre du Deutéronome met Israël en garde en ces termes : «Tu n'auras pas en abomination l'Édomite, car il est ton frère ( $k i$ ahikha hu) » (Dt 23, 8). À l'inverse, l'Ammonite et le Moabite sont radicalement condamnés et exclus à jamais de l'assemblée d'Israël pour n'être pas venus au-devant des Israélites avec de l'eau et du pain après la sortie d'Égypte, et pour avoir mobilisé contre Israël le devin Balaam (Dt 23, 3-6). Peut-être est-ce également dû au fait que leur parenté avec Israël est plus lointaine et ne remonte pas à Abraham lui-même, mais seulement aux ancêtres de ce dernier.

Plusieurs passages remarquables concernant le lien de fraternité entre Israël et Édom se rencontrent dans les livres prophétiques. On lit ainsi dans le Livre d'Amos : «Ainsi parle le Seigneur : À cause de trois transgressions d'Édom, à cause de quatre, je ne révoquerai pas mon arrêt: parce qu'il a poursuivi son frère avec l'épée en étouffant sa compassion, parce que sa colère déchire sans cesse et qu'il garde continuellement sa fureur $[\ldots] »($ Am 1, 11). Le motif revient avec insistance dans le Livre d'Abdias, dirigé entièrement contre Édom : «À cause des violences exercées contre Jacob, ton frère, tu seras couvert de honte et tu seras retranché pour toujours » $(\mathrm{Ab} 10)$; «Ne prends pas plaisir à voir le jour de ton frère, le jour de son infortune » (Ab 12). Le rejet d'Ésaü - Édom est encore plus radical dans le Livre de Malachie :

Je vous ai aimés, dit YHWH, mais vous dites : «En quoi nous aimes-tu? ». Ésaü n'est-il pas frère de Jacob? - déclaration de YHWH. Cependant j'ai aimé Jacob et j'ai détesté Ésaü : j'ai fait de ses montagnes un lieu dévasté, j'ai livré son patrimoine aux chacals du désert. Si Édom dit : «Nous avons été détruits, mais nous rebâtirons les ruines !»-ainsi parle YHWH des armées: Qu'ils bâtissent, je raserai! On les appellera «Territoire de la méchanceté », "Peuple contre lequel YHWH est furieux pour toujours ». (Mal 1, 2-4) 
Même si ce dernier texte semble la réduire à néant, l'idée d'une fraternité entre Israélites et Édomites dont la validité perdure pardelà les générations, depuis Jacob et Ésaü, est frappante et, à ma connaissance, unique dans le corpus de la Bible hébraïque. Même dans le cas d'Ismaël, le demi-frère d'Isaac, descendant direct d'Abraham, on ne rencontre pas d'équivalent.

\section{LE JUDAÏSME ET LES SYNGENEIAI DU MONDE HELLÉNISTIQUE}

Dans le monde hellénistique, les parentés mythiques ou légendaires reliant des poleis ou des peuples étaient fréquemment invoquées dans le contexte d'échanges diplomatiques. Ce langage de la parenté comportait une dimension rhétorique importante, mais avait aussi des conséquences politiques concrètes ${ }^{12}$. Dans tous les cas, les Grecs étaient attentifs à ces formes de parenté, et pouvaient argumenter avec soin, à partir de récits mythologiques, épiques ou historiographiques, afin de démontrer une origine commune avec une autre cité et l'existence d'une syngeneia. Les Juifs vivant au sein du monde hellénistique adoptèrent également ce modèle culturel et inventèrent de nouveaux types de parenté forgés sur le modèle des syngeneiai hellénistiques, qui vinrent s'ajouter à ceux déjà véhiculés par les textes bibliques.

Examinons tout d'abord quelles furent les évolutions dans les représentations des liens de parenté hérités de la tradition biblique. Dans la plupart des cas, les peuples installés dans les régions avoisinant la Judée furent identifiés aux nations bibliques (Édomites, Moabites, Ammonites, etc.). Ainsi la Septante rendelle Dt 23, 8 par : «Tu n'auras pas en horreur un Iduméen, car il

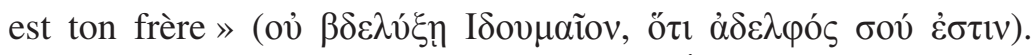
Là où le texte hébraïque utilise le mot «Édomite», la Septante a «Iduméen», ce qui montre que les Iduméens de l'époque hellénistique étaient perçus comme le même groupe ethnique que les Édomites, indépendamment de leur déplacement géographique de la Transjordanie vers la partie méridionale de la Judée et le nord

12. Voir Olivier Curty, Les parentés légendaires entre cités grecques, Paris, Droz, 1995, p. 254-258 en particulier. 
du Néguev ${ }^{13}$. Par la suite, ils seront intégrés à la Judée sous Jean Hyrcan ; Josèphe rapporte que ce dernier leur imposa la pratique de la circoncision (en réalité déjà largement pratiquée en contexte iduméen) et les lois judéennes (A.J. XIII, 257-258). Certains ont avancé que ce fut la parenté des Iduméens - Édomites avec les Judéens descendants de Jacob qui inspira la politique d'Hyrcan Ir, mais aucune source ne le confirme explicitement ${ }^{14}$. En lien avec la période hasmonéenne, Josèphe note dans les Antiquités que les Iduméens sont des « descendants d'Ésaü » (A.J. XII, 328). Comme la Septante, Josèphe identifie par conséquent les Iduméens avec les Édomites bibliques, suggérant par là qu'il existait un lien de parenté entre les Judéens et les Iduméens. Pourtant, lorsqu'il évoque la judaïsation des Iduméens sous Jean Hyrcan, Josèphe ne mentionne pas ce lien de parenté ${ }^{15}$.

Il n'est pas sans intérêt de relever qu'il y eut au moins un auteur grec, en la personne d'Alexandre Polyhistor au I $^{\text {er }}$ siècle av. n. è., pour relever cette proximité entre Judéens et Iduméens. Il semble l'avoir justifiée non par une référence au récit biblique, mais par une parenté mythique remontant à la légendaire reine de Babylone, Sémiramis. On lit en effet chez Étienne de Byzance ( $\mathrm{VI}^{\mathrm{e}}$ siècle) : «Judée. Alexandre Polyhistor (dit que le nom dérive de ceux) des

13. Sur cette évolution historique, voir A. Lemaire, « D'Édom à l'Idumée et à Rome », p. 81-96.

14. Voir Morton Smith, "The Gentiles in Judaism, 125 BCE - CE 66 », The Cambridge History of Judaism. III. The Early Roman Period, dir. William Horbury, William D. Davies et John Sturdy, Cambridge, Cambridge University Press, 1999, p. 192-249 (en particulier p. 208).

15. Sur la judaïsation des Iduméens, la bibliographie est abondante. Voir entre autres Aryeh Kasher, Jews, Idumaeans, and Ancient Arabs: Relations of the Jews in Eretz-Israel with the Nations of the Frontier and the Desert during the Hellenistic and Roman era (332 BCE-70 CE), Tübingen, Mohr Siebeck, 1988 ; Richard A. Horsley, "The Expansion of Hasmonean Rule in Idumea and Galilee: Toward a Historical Sociology », Second Temple Studies III. Studies in Politics, Class and Material Culture, dir. R. Davies et John M. Halligan, Sheffield, Sheffield Academic Press, 2002, p. 134-165 ; Seth Schwartz, « Conversion to Judaism in the Second Temple Period: A Functionalist Approach », Studies in Josephus and the Varieties of Ancient Judaism: Louis H. Feldman Jubilees Volume, dir. Shaye J. D. Cohen et Joshua J. Schwartz, Leiden, Brill, 2007, p. 223-236 ; Uriel Rappaport, « La judaïsation des Iduméens à l'époque de Jean Hyrcan », Israel's Land: Papers Presented to Israel Shatzman on his Jubilee, dir. Joseph Geiger, Hannah M. Cotton et Guy Stiebel, Raanana, The Open University of Israel, 2009, p. 59-74 (en hébreu). 
enfants de Sémiramis, Juda et Idumée $»^{16}$. Shaye Cohen note à cet égard : « Like many other ethnographers, Polyhistor did not hesitate to employ fanciful etymologies to explain exotic geographic names. Thus the name "Judaea" is said to derive from two eponymous heroes, Juda and Idumaea »- lesquels sont présentés comme des frères. Pour Cohen, Polyhistor pourrait se faire l'écho d'une période d'alliance entre Judéens et Iduméens : «Since this fragment has no hint of any rivalry or hostility between the brothers, it seems to suggest that Judaea is the peaceful agglomeration of Judaeans and Idumaeans $»^{17}$. On pense évidemment à la période ayant suivi la conquête de l'Idumée par Hyrcan, au $\mathrm{I}^{\text {er }}$ siècle av. n. è., qui vit un descendant d'Iduméen, Hérode, s'emparer du trône de Judée.

Dans les textes juifs d'époque hellénistique figurent d'autres échos des parentés bibliques, en lien avec des peuples contemporains des Judéens. Les Arabes, en particulier, sont identifiés avec les descendants d'Ismaël ${ }^{18}$. Selon Artapan, un auteur juif écrivant en grec au $\mathrm{III}^{\mathrm{e}}$ ou au $\mathrm{II}^{\mathrm{e}}$ siècle av. n. è., les Ismaélites à qui Juda et ses frères vendirent Joseph (d'après Gn 37, 25) étaient des « Arabes » ${ }^{19}$. Dans le Livre des Jubilés, rédigé en hébreu au II $^{\mathrm{e}}$ siècle av. n. è., on lit à propos de la décision d'Abraham d'éloigner Ismaël d'Isaac :

« Ismaël et ses fils, les fils de Qetourah et leurs fils s'en allèrent ensemble. Ils s'établirent depuis Paran jusqu'à l'entrée de Babylone,

16. Voir Menahem Stern, Greek and Latin Authors on Jews and Judaism, Jerusalem, Israel Academy of Sciences and Humanities, 1974, vol. I, p. 164 (n53).

17. Shaye Cohen, The Beginnings of Jewishness: Boundaries, Varieties, Uncertainties, Berkeley, University of California Press, 1999, p. 113.

18. Voir Israel Eph'al, "Ishmael and 'Arab(s)': A Transformation of Ethnological Terms », Journal of Near Eastern Studies 35, 1976, p. 225-231 ; Fergus Millar, "Hagar, Ishmael, Josephus and the Origins of Islam », Journal of Jewish Studies 44/1, 1993, p. 23-45 ; Erich S. Gruen, « Kinship Relations and Jewish Identity », Jewish Identities in Antiquity: Studies in Memory of Menahem Stern, dir. Lee I. Levine et Daniel R. Schwartz, Tübingen, Mohr Siebeck, 2009, p. 101-116.

19. Artapan réécrit librement le récit de la Genèse, puisqu'il dit que Joseph s'enfuit et demanda lui-même aux Arabes de l'emmener en Égypte. Il écrit que « les rois des Arabes sont descendants d'Israël, des fils d'Abraham, des frères d'Isaac » (Eusèbe, Préparation Évangélique (P.E.) IX.23.1). Son œuvre est citée par Eusèbe, mais là encore par l'intermédiaire d'Alexandre Polyhistor, ce qui pourrait expliquer certaines erreurs (tel le fait qu'Israël semble présenté comme le père d'Abraham plutôt que comme son petit-fils). Sur Artapan, voir l'ouvrage récent dirigé par Philippe Borgeaud, Thomas Römer et Youri Volokhine, Interprétations de Moïse : Égypte, Judée, Grèce et Rome, Leiden, Brill, 2010, p. 3-82. 
dans tout le pays qui est du côté de l'orient, en face du désert. Ils se mêlèrent les uns aux autres, et ils furent appelés Arabes et Ismaélites » (Jubilés XX, 12-13 $)^{20}$.

Le nom «Arabes» est visiblement compris par l'auteur des Jubilés comme dérivant de la racine 'arav, "mélanger »; surtout, il est rattaché aux descendants d'Ismaël. À la fin du $\mathrm{I}^{\mathrm{er}}$ siècle de n. è., l'identification des Arabes avec les Ismaélites allait de soi pour Josèphe ${ }^{21}$. Cette tradition était même connue d'un auteur grec hostile aux Juifs, le rhéteur Apollonios Molon ( $\mathrm{I}^{\mathrm{er}}$ siècle de n. è.), qui la modifie toutefois en attribuant à Hagar non pas un, mais douze fils, qui deviennent par la suite les douze rois des Arabes ${ }^{22}$.

Enfin, Josèphe mentionne également une parenté entre les Juifs et les Chaldéens, sans doute inspirée du récit des origines chaldéennes

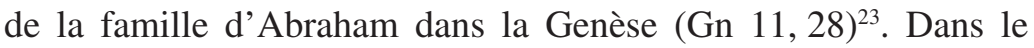
Contre Apion, il écrit au sujet des Chaldéens : «(...) ils furent les ancêtres de notre race (genos) et, à cause de cette parenté (dia tèn syngeneian), ils mentionnent les Juifs dans leurs annales » (I, 71) ${ }^{24}$. Le but de Josèphe est de prouver l'ancienneté du peuple juif en s'appuyant sur le témoignage d'auteurs issus des peuples voisins ; il reste difficile de savoir à quels textes il fait allusion ${ }^{25}$.

Outre ces parentés inspirées de la Bible et actualisées par les auteurs juifs de l'époque hellénistique et romaine, de nouveaux types de parentés apparaissent dans les textes juifs, en adéquation avec le modèle des syngeneiai hellénistiques. Des auteurs juifs imaginent des liens de parenté entre les Juifs et des peuples qui

20. Trad. A. Caquot, La Bible. Écrits intertestamentaires, dir. André DupontSommer et Marc Philonenko, Paris, Gallimard (Pléiade), 1987, p. 716.

21. A.J. I, 220-221; I, 239; II, 213. Comme le remarque Erich Gruen, « The literary appropriation of Arabs for Abraham's line in the Hellenistic period further illustrates that powerful propension for incorporating the outsider by making him a kinsman » (E. Gruen, « Kinship Relations », p. 114).

22. Voir Eusèbe, P.E. IX.19.2.

23. Cf. John Barclay, Josephus. Against Apion, Leiden, Brill, 2009, p. 47, note 283: "Following the LXX, Josephus takes Abraham's ancestry to derive from "Chaldea" (Gen 11:28; Ant. 1.151-68)».

24. Trad. Léon Blum, Flavius Josèphe. Contre Apion, Paris, Les Belles Lettres,

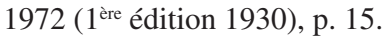

25. Cf. J. Barclay, Josephus. Against Apion, p. 47, note 284 : « As far as we can tell, no "Chaldean" source ever recognizes Judeans as a kindred people, and this is certainly not hinted at in the material from Berosus to be cited: in 1.137 (the only reference to Judeans), they are listed among other captives, with indifference ». 
n'étaient nullement mentionnés dans la Bible, en postulant des ancêtres communs situés dans un temps très éloigné.

Parmi les fragments d'auteurs juifs hellénistiques cités par Alexandre Polyhistor et préservés dans la Préparation évangélique d'Eusèbe, on trouve un récit attribué à un certain Cléodème Malchos. En lien avec Genèse 25, 1-6, qui énumère les descendants issus de l'union d'Abraham avec Qetourah, Cléodème rapporte le mariage d'Héraclès avec l'une des petites-filles d'Abraham ${ }^{26}$. Les descendants d'Héraclès (ceux du moins qui naquirent de cette union) étaient par conséquent des descendants d'Abraham, de sorte qu'il devenait envisageable que certains Grecs qui se rattachaient à la figure tutélaire d'Héraclès soient de lointains cousins des Juifs. On notera toutefois que, tant d'après Josèphe que d'après Eusèbe - ce qui signifie que ce détail remonte au moins à Polyhistor -, le récit de Cléodème ne sert pas vraiment le rapprochement entre Juifs et Grecs. Certes, Héraclès épouse la fille d'Aphra, l'un des fils d'Abraham et Qetourah, et il a d'elle un fils nommé Didôros, duquel naît Sophôn. Mais Josèphe ajoute alors : "C'est de lui que les Barbares tiennent le nom de Sophaques » (A.J. I, 241), ce qui laisse plutôt entendre que l'union d'Héraclès et de la petite-fille d'Abraham déboucha sur la formation d'un nouveau peuple barbare ${ }^{27}$. On pouvait toutefois imaginer que d'autres enfants étaient nés de l'union d'Héraclès avec la fille d'Aphra... Par ailleurs Cléodème présente également les Assyriens et les peuples d'Afrique comme des descendants d'Abraham et Qetourah, en opposition avec les généalogies bibliques, qui voyaient dans les Africains des descendants de Ham, le fils de Noé et le frère de Shem. Erich Gruen qualifie ce récit de « mishmash of biblical genealogy, Greek legend, and Jewish fiction ». Surtout, il souligne à juste titre que le texte de Cléodème « does not constitute a Jewish effort at assimilation. Rather, the labors of Herakles and the African succession are brought into line on behalf of the heritage of Abraham. [...] The outsider is brought inside $»^{28}$.

26. Voir Eusèbe, P.E. IX.20.2-4, ainsi que Josèphe, A.J. I, 239-241 ; l'un et l'autre précisent que l'installation des descendants d'Abraham en Afrique commença par la Lybie.

27. De même, chez Eusèbe, on lit : «Héraclès épousa la fille d'Aphra et en eut un fils, Diodoros ; celui-ci engendra Sophonas, qui a fait appeler ces barbares Sophaques » (P.E. IX.20.4, trad. Édouard des Places, SC 369, Paris, Cerf, 1991, p. 247).

28. E. Gruen, « Kinship Relations », p. 109-110. 
Cette conclusion s'applique également à une autre parenté fictive inventée par des Juifs à l'époque hellénistique, celle entre les Juifs et les Spartiates, dont nous trouvons la trace dans les deux premiers Livres des Maccabées. En lien avec la période précédant la crise maccabéenne, le Deuxième Livre des Maccabées (5, 6-10) rapporte que le grand prêtre Jason, destitué, chercha à se réfugier à Sparte, au nom de la parenté entre Juifs et Spartiates (dia tèn syngeneian, §9). Jan Bremmer rapproche le nom du grand prêtre ayant succédé à Jason, Ménélas, de celui du roi de Sparte durant la guerre de Troie, et y voit un autre signe de la popularité, parmi les élites hellénisées de Jérusalem, de cette parenté fictive entre Spartiates et Juifs ${ }^{29}$. Mais c'est le Premier Livre des Maccabées qui va le plus loin dans l'exposé des relations entre les deux peuples, en citant des lettres (fictives) qui témoigneraient d'échanges diplomatiques entre Sparte et Jérusalem. Dans l'ordre chronologique supposé des lettres, vient tout d'abord une lettre du roi de Sparte Areus (309/8-265 av. n. è.) adressée au grand prêtre Onias (sans doute Onias $\mathrm{I}^{\mathrm{er}}$ ), rapportant la découverte à Sparte d'un document prouvant que les Juifs et les Spartiates sont «frères » (adelphoi), « de la lignée d'Abraham »

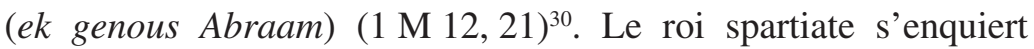
alors de l'état des affaires des Juifs. Dans ce même chapitre 12 figure la réponse envoyée par Jonathan Maccabée près d'un siècle plus tard, dans laquelle il exprime le désir des Juifs de Judée de renouveler «(les liens de) fraternité et (d') amitié », afin de ne pas devenir des étrangers les uns pour les autres $(1 \mathrm{M} \mathrm{12,10).}$ Dans le passage parallèle des Antiquités juives (XIII, 164-170), Josèphe écrit qu'ils voulaient rappeler aux Spartiates «l'amitié et la parenté » (philian kai syngeneian) des Judéens avec eux. La troisième pièce de l'échange épistolaire judéo-spartiate est citée dans 1 Maccabées 14, 16-23 : il s'agit de la réponse des Spartiates à la lettre de Jonathan (désormais décédé, mais remplacé par son frère Simon), dans laquelle ils expriment leur désir de renouveler l'amitié (philia) avec les Judéens.

29. Jan Bremmer, «Spartans and Jews: Abrahamic Cousins? », Abraham, the Nations, and the Hagarites, dir. Martin Goodman, George van Kooten et Jacques van Ruiten, Leiden, Brill, 2010, p. 47-59.

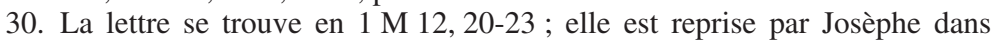
A.J. XII, 225-227. 
Le cas spartiate, qui représente l'attestation la plus claire de l'invention d'une parenté entre Juifs et Grecs, est d'autant plus intéressant qu'il apparaît dans un ouvrage rédigé en hébreu à la cour hasmonéenne (1 Maccabées), et qu'il implique une utilisation politique de ladite parenté, ne serait-ce que dans un but de propagande interne ${ }^{31}$. Il est probable que l'image des Spartiates comme un peuple courageux, discipliné, au mode de vie austère, de grande valeur militaire et fidèle à ses lois ancestrales ait joué un rôle dans la volonté de certains Juifs de revendiquer une parenté avec les Spartiates (même si la Sparte du milieu du II $^{\mathrm{e}}$ siècle av. n. è. était très éloignée de sa grandeur passée) $)^{32}$. Le témoignage de Josèphe dans le Contre Apion, à la fin du $\mathrm{I}^{\mathrm{er}}$ siècle, corrobore en tout cas cette hypothèse, car Josèphe déploie une stratégie apologétique qui s'appuie précisément sur ces topoi ${ }^{33}$. Néanmoins, comme le note justement Erich Gruen, la parenté avec les Spartiates est conçue selon un modèle juif, puisque c'est Abraham l'ancêtre commun, et non quelque figure mise en avant par les Spartiates eux-mêmes. Une fois de plus, «the outsider is brought inside », dans le cadre d'une vision du monde qui reste judéocentrée ${ }^{34}$. Il est possible que

31. Tout comme Erich Gruen et Jan Bremmer, je doute de la réalité historique de l'échange épistolaire et diplomatique mentionné dans 1 Maccabées.

32. Voir François Ollier, Le mirage spartiate: Étude sur l'idéalisation de Sparte dans l'Antiquité grecque du début de l'école cynique jusqu'à la fin de la cité, Paris, De Boccard, 1933-43 (2 vols.) ; Olivier Curty, « À propos de la parenté entre Juifs et Spartiates », L'étranger dans le monde grec: Actes du [second] colloque, Nancy, mai 1987, dir. Raoul Lonis, Nancy, Presses universitaires de Nancy, 1988, p. 169-191; Erich S. Gruen, «The Purported Jewish-Spartan Affiliation », Transitions to Empire: Essays in Greco-Roman History, 300-146 B.C. in Honor of E. Badian, dir. Robert W. Wallace et Edward M. Harris, Norman, Univ. of Oklahoma Press, 1996, p. 254-269; id., « Kinship Relations », p. 111 ( « The value of an association with Sparta for Jewish self-esteem in a Greek world is plain enough »);id., Rethinking the Other in Antiquity, Princeton, Princeton University Press, 2010, p. 305-306.

33. Voir Contre Apion II, 225-235. Il est probable que dans le contexte hasmonéen, d'autres facteurs aient joué, mais je ne peux les présenter ici.

34. Il faut par conséquent nuancer l'affirmation de Martin Goodman, qui compare la parenté judéo-spartiate à la revendication d'une origine troyenne par les Romains et écrit : " Neither Jews nor Romans were unusual in the Mediterranean world in their search into Greek prehistory for such links to their origins. Many peoples besides the Romans claimed a link to Troy, and claims of Spartan ancestry were quite common» (Rome and Jerusalem, New York, A. A. Knopf, 2007, p. 104). En réalité les Judéens ne revendiquèrent pas une ascendance spartiate, mais présentèrent les Lacédémoniens comme ayant un ancêtre "juif" (pour autant que l'on puisse appliquer le terme à Abraham, bien sûr). La différence est essentielle. 
le lien entre Abraham et les Spartiates ait été conçu à partir de récits associant Héraclès et Abraham, comme celui attribué à Cléodème Malchos; les rois de Sparte se présentaient en effet comme les descendants des Héraclides, eux-mêmes descendants d'Héraclès. Mais les Livres des Maccabées ne le précisent pas.

En bref, dans un monde hellénistique rempli de syngeneiai diverses et variées, les Juifs ne furent pas en reste. Les parentés bibliques furent revisitées et actualisées, tandis que de nouveaux types de parenté étaient imaginés. Ces dernières se caractérisaient par le choix de faire d'Abraham l' ancêtre commun aux Juifs et aux autres peuples. Noé, Shem ou d'autres figures précédant Abraham auraient pu être invoquées, mais elles n'étaient probablement pas assez spécifiques, puisque l'histoire d'Israël stricto sensu ne commence qu'avec Abraham. Surtout, la bénédiction prononcée sur Abraham, selon laquelle il deviendrait le père de nombreuses nations (Gn 17,4), ouvrait la voie au rattachement de bien des peuples à la figure du patriarche. Ces généalogies et parentés fictives permirent aux Juifs de dessiner une carte mentale du monde où chaque peuple recevait une place en fonction de sa proximité ou de son éloignement avec Israël. Or assigner une place à quelqu'un correspond à une position de pouvoir, que celui-ci soit réel ou fictif, concret ou symbolique. Il faudra nous en souvenir pour évaluer la portée de l'invention de la fraternité judéo-romaine : l'identification de Rome avec Ésaü - Édom doit sans doute aussi être appréciée à la lumière de l'absence de pouvoir expérimentée par les Juifs face à Rome, et comprise comme une forme de compensation liée à la maîtrise imaginaire, mentale, de celui sur qui on n'a aucune prise.

\section{LA FRATERNITÉ ENTRE JACOB - ISRAËL ET ÉSAÜ - ROME}

\section{L'identification de Rome avec Ésaï - Édom : un autre cas de syngeneia mythique?}

Rome commence à apparaître dans les sources juives d'époque hellénistique à la suite du conflit maccabéen. Le Premier Livre des Maccabées rapporte des échanges diplomatiques entre la dynastie hasmonéenne et les Romains, désignés dans la traduction grecque 
par le terme Rômaioi ${ }^{35}$, tandis que le Livre de Daniel, le Rouleau de la Guerre et certains pesharim de Qumrân utilisent le terme hébraïque Kittim, qui à l'origine renvoyait aux Grecs, descendants de Japhet (Gn 10, 4), et dans certains cas, plus spécifiquement, aux Séleucides $^{36}$. Ce lien entre Romains et Grecs est logique, puisque les Romains sont des "Occidentaux" relevant globalement du territoire de Japhet. La perception originelle des Romains par les Juifs n'implique par conséquent aucune parenté entre les deux peuples, sauf à remonter à Noé, père de l'humanité postdiluvienne dans son ensemble.

Certains textes juifs vont également proposer des typologies, en identifiant Rome avec le quatrième empire évoqué dans le Livre de Daniel, avec Tyr ou encore avec Babylone ${ }^{37}$. Le choix de Babylone, attesté surtout dans les apocalypses ${ }^{38}$, apparaît particulièrement pertinent. En effet, Babylone et Rome étaient toutes les deux des villes; les Babyloniens avaient causé la destruction du premier temple, tandis que les Romains avaient détruit le second ${ }^{39}$; les

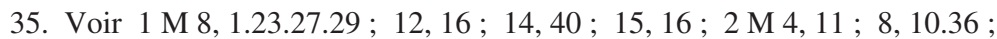
11,34 .

36. Voir Daniel 11, 30 (le texte massorétique a Kittim, que la Septante traduit par Rômaioi) ; Rouleau de la Guerre (1QM) XIX 10 (entre autres) ; Pesher Nahum (4Q169) 3-4 i 3 ; Pesher Habaquq II 12.14.16. Sur Rome dans les textes de Qumrân, voir Philip Alexander, «The Evil Empire: The Qumran Eschatological War Cycle and the Origins of Jewish Opposition to Rome », Emanuel. Studies in Hebrew Bible, Septuagint and Dead Sea Scrolls in Honor of Emanuel Tov, dir. Paul Shalom et al., Leiden, Brill, 2003, p. 17-31 ; Brian Schultz, « Not Greeks but Romans: Changing Expectations for the Eschatological War in the War Texts from Qumran », The Jewish Revolt against Rome: Interdisciplinary Perspectives, dir. Mladen Popović, Leiden, Brill, 2011, p. 107-127 ; Kenneth Atkinson, « Historical References and Allusions to Foreigners in the Dead Sea Scrolls: Seleucids, Ptolemies, Nabateans, Itureans, and Romans in the Qumran Corpus », The Qumran Chronicle 21/1-2, 2013, p. 1-32.

37. Voir Mireille Hadas-Lebel, Jérusalem contre Rome, Paris, Éditions du Cerf, 1990, p. 389-405, 430-435. Voir également le rappel de ces identifications successives dans l'article de Hervé Inglebert dans le présent dossier.

38. Voir IV Esdras III, 28.32; XV, 43-48; XVI, 1; II Baruch XI, 1-2; LXVII, 7-8 ; Apocalypse de Jean 14, 8 ; 16, 19 ; 17, 5; 18, 2.10 .21 (et aussi $1 \mathrm{P} 5,13)$.

39. Flavius Josèphe reproduit une tradition selon laquelle les destructions du premier et du second temple eurent toutes deux lieu un 9 du mois de Av, ce qui suggère un parallélisme entre les destructeurs, Babylone et Rome. De plus il n'est pas anodin que Flavius Josèphe se soit perçu comme un nouveau Jérémie : cela crée une relation typologique entre les deux prises de Jérusalem, ainsi qu'entre Rome et Babylone. Cf. Mireille Hadas-Lebel, « Flavius Josèphe entre Polybe et Jérémie », Ktema 24, 1999, p. 159-165. 
Babyloniens déportèrent une partie de la population du royaume de Juda, tout comme les Romains emmenèrent de nombreux Judéens captifs en Italie ; Babylone avait été la tête d'un empire, tout comme Rome l'était au $\mathrm{I}^{\mathrm{er}}$ siècle de n. è. Enfin, les nombreux oracles de châtiment contre Babylone contenus dans les textes bibliques pouvaient aisément être reportés sur Rome pour exhorter et consoler les Judéens vaincus. Cependant, comme le rappelle Mireille HadasLebel, le schéma de la succession des empires et le rôle de « dernier empire » dévolu à Rome dans celui-ci, empêchaient d'identifier purement et simplement Rome et Babylone ${ }^{40}$. Hervé Inglebert note en outre à juste titre que le développement du judaïsme rabbinique en Babylonie devait rendre l'identification de Rome avec Babylone insatisfaisante aux yeux des Sages ${ }^{41}$.

Dans la littérature rabbinique, Rome, le « royaume impie», est associée de manière croissante avec Édom et Ésaü. Cette association, prise au premier degré, implique l'existence d'un lien de parenté entre Juifs et Romains. Or une telle parenté est problématique à bien des égards. D'une part, elle contredit le rattachement traditionnel des Romains aux descendants de Japhet, en en faisant des Sémites, descendants d'Abraham (même si dans l'absolu il y avait moyen de contourner cette difficulté en supposant, comme dans le récit de Cléodème Malchos, une union entre une descendante d'Abraham et un ancêtre d'Énée, par exemple). D'autre part, l'association entre Rome et Ésaü - Édom diffère des syngeneiai d'époque hellénistique comme celle entre les Juifs et les Spartiates, dans la mesure où elle assimile les Romains à un peuple déjà identifié dans la Bible comme un voisin historique d'Israël au Proche-Orient. S'il était compréhensible, au nom d'une certaine continuité géographique et ethnique, de rapprocher Édomites et Iduméens ou encore Ismaélites et Arabes, le rapprochement entre Édomites et Romains était pour le moins osé. Il l'était d'autant plus qu'une bonne partie des Iduméens avaient été intégrés à l'ethnos judéen sous les Hasmonéens, au point de donner à la Judée une dynastie de rois, en la personne d'Hérode et de ses descendants ${ }^{42}$. Cette intégration eut des effets durables, puisque l'on vit des Iduméens combattre les Romains aux côtés

40. M. Hadas-Lebel, Jérusalem contre Rome, p. 433.

41. Voir son article dans ce dossier.

42. Comme le souligne justement Friedrich Avemarie dans «Esaus Hände, Jakobs Stimme », p. 178. Sur la judaïsation des Iduméens, voir supra, note 15. 
des Judéens lors de la guerre de 66-70, un fait que les rabbins de la fin du $\mathrm{I}^{\mathrm{er}}$ siècle et du début du II $^{\mathrm{e}}$ siècle de notre ère pouvaient difficilement ignorer ${ }^{43}$.

L'identification de Rome avec Ésaü - Édom ne relève donc pas de la création d'une nouvelle syngeneia entre Israël et un autre peuple, mais de l'établissement d'une typologie. Cependant, cette typologie même est lourde d'implications : prise au premier degré, de manière littérale, elle signifie qu'il existe un lien de parenté et même une fraternité entre Israël et Rome, une idée qui parut peutêtre scandaleuse ou absurde à certains Juifs. Aucun texte juif de l'Antiquité ne semble soucieux d'expliquer quels pouvaient être les fondements historiques d'une telle parenté, mais on connaît un auteur juif médiéval qui ressentit le besoin de combler cette lacune et entreprit de fonder l'existence d'une parenté entre Juifs et Romains sur un récit conforme aux normes gréco-romaines de vraisemblance et d'historicité en matière de syngeneiai.

Ce récit se trouve dans le Sefer Yosippon, un ouvrage rédigé en hébreu par un Juif d'Italie méridionale au cours du $\mathrm{x}^{\mathrm{e}}$ siècle (les spécialistes avancent la date de 953), et qui se présente comme une histoire du judaïsme durant la période du Second Temple. Son contenu a pu être présenté comme « the essentials of Livy and Virgil [...] set forth in Hebrew for the first time from a Jewish point of view $»^{44}$. Le Sefer Yosippon, qui hérite de toute évidence de la tradition identifiant Rome à Ésaü - Édom, se pose la question du lien généalogique entre les descendants d'Ésaü et les Romains de la période du Second Temple, et propose le scénario historique suivant, fondé sur l'idée d'une migration édomite vers Rome : le petit-fils d'Ésaü, un certain Zepho, se rendit à Carthage, où il entra au service d'Énée, avant de rejoindre les Kittim d'Italie et de devenir leur roi. Par la suite, un de ses descendants fut Romulus. Durant le règne de ce dernier, des Édomites se réfugièrent à Rome pour échapper au roi David, et s'y installèrent. Le lien entre Rome et Ésaü - Édom est

43. Voir B.J. IV, 224.229.566-576; V, 248-249; VI, 378-383; Alan Appelbaum, "The Idumaeans" in Josephus' The Jewish War», Journal for the Study of Judaism 40/1, 2009, p. 1-22; Adam Kolman Marshak, «Rise of the Idumeans: Ethnicity and Politics in Herod's Judea », Jewish Identity and Politics Between the Maccabees and Bar Kokhba: Groups, Normativity, and Rituals, dir. Benedikt Eckhardt, Leiden, Brill, 2012, p. 117-129.

44. G. Cohen, «Esau as Symbol», p. 40. 
donc double : il se situe au niveau d'une généalogie commune via Zepho, qui fait d'Ésaü l'ancêtre lointain de Romulus, mais il repose aussi sur l'installation d'Édomites à Rome, dès les origines, ce qui permet d'envisager qu'une partie des Romains au moins aient eu des origines édomites, et non le seul Romulus. Pour Joshua Holo, le Sefer Yosippon « validates, in other words, the rabbis' ethnography of Rome by attending to Greco-Roman standards of historical proof $»^{45}$. Mais il s'agit plutôt de typologie que d'ethnographie! Car en ce qui concerne la tradition rabbinique elle-même, elle ne fournit justement aucune donnée généalogique ou ethnographique permettant de préciser la nature du lien entre Juifs et Romains. On reste bel et bien au niveau de la typologie.

\section{L'origine et la signification de l'identification de Rome avec Ésaü - Édom}

Comme je l'ai rappelé dans l'introduction, pour certains chercheurs l'identification de Rome avec le frère jumeau d'Israël serait intimement liée à la christianisation de l'empire et daterait au mieux de la fin du IV siècle. À l'inverse, d'autres historiens estiment que cette identification remonte à la destruction du Temple en 70 , ou encore à la période de la révolte de Bar Kokhba ${ }^{46}$. Plusieurs arguments permettent me semble-t-il de privilégier une datation de l'identification de Rome avec Édom - Ésaü en lien avec l'échec des révoltes contre Rome, dès la fin du $\mathrm{I}^{\text {er }}$ siècle ou le $\mathrm{II}^{\mathrm{e}}$ siècle de $\mathrm{n}$. è.

La tradition apocalyptique fournit un premier indice. Dans le $I V^{e}$ Livre d'Esdras, le narrateur questionne son interlocuteur angélique au sujet de la fin des temps et de ses divisions :

45. Joshua Holo, «Byzantine-Jewish Ethnography: A Consideration of the "Sefer Yosippon" in light of Gerson Cohen's "Esau as Symbol in Early Medieval Thought" ", Jews in Byzantium: Dialectics of Minority and Majority Cultures, dir. Robert Bonfil et al., Leiden, Brill, 2012, p. 923-949, citation p. 924.

46. Voir entre autres George Foot Moore, Judaism in the First Centuries of the Christian Era, the Age of the Tannaim, New York, Schocken Books, 1971, vol. II, p. 116 et 329 ; G. Cohen, «Esau as Symbol »; M. Hadas-Lebel, Jérusalem contre Rome, p. 461-465 ; F. Avemarie, «Esaus Hände, Jakobs Stimme », p. 180-184 ; Israel Yuval, Two nations in Your Womb: Perceptions of Jews and Christians in Late Antiquity and the Middle Ages, trad. Barbara Harshav et Jonathan Chipman, Berkeley, University of California Press, 2006, p. 10-11; Adiel Schremer, Brothers Estranged: Heresy, Christianity, and Jewish Identity in Late Antiquity, New York, Oxford University Press, 2010, p. 131-134 et note 61, p. 227. 
Je répondis et dis : «Quelle sera la division des temps ? Quand viendra la fin du premier et le début du suivant?»Et il me dit: «D'Abraham à Abraham. De lui naquirent Jacob et Ésaü. La main de Jacob tenait au commencement le talon d'Ésaü. La fin de ce siècle, c'est Ésaü. Le commencement du suivant, c'est Jacob. Car la main c'est le principe de l'homme, et le talon c'est sa fin. Entre le talon et la main, ne cherche rien d'autre, Esdras. ${ }^{47}$

Les spécialistes du IVe Livre d'Esdras s'accordent à dater l'ouvrage de la fin du $\mathrm{I}^{\mathrm{er}}$ siècle de $\mathrm{n}$. è. et à voir dans les visions d'Esdras et son dialogue avec l'ange, une réponse au désespoir d'au moins une partie des Juifs après la destruction du Temple et le triomphe de Rome ${ }^{48}$. Il est donc quasiment certain qu' «Ésaü » renvoie ici à l'empire romain, dont la domination prendra fin pour céder la place au règne d'Israël dans « l'âge qui vient ${ }^{49}$. L'image de la main de Jacob tenant le talon d'Ésaü permet en outre à l'auteur d'affirmer que le triomphe d'Israël succédera immédiatement à la fin de Rome, sans qu'un autre royaume ne fasse irruption et ne vienne s'immiscer entre les deux.

Un autre indice provient de l'œuvre de Flavius Josèphe, plus ou moins contemporaine du IV Livre d'Esdras. Au livre IV, Josèphe réécrit l'épisode biblique de Balaam, le devin invité par le roi de Moab à maudire Israël et qui prophétise à l'inverse le triomphe du peuple aimé de Dieu. Particulièrement important, à cet égard, est l'oracle contenu en Nombres 24, 17-19:

$17 \mathrm{Je}$ le vois, mais non maintenant. Je le contemple, mais non de près. Un astre sort de Jacob, un sceptre s'élève d'Israël. Il fracasse les tempes de Moab, et il détruit tous les enfants de Seth. 18 Édom sera pays conquis, Séir - ses ennemis - sera pays conquis. Israël manifeste

47. IV Esdras VI, 7-10, traduction de Pierre Geoltrain, La Bible. Écrit intertestamentaires, p. 1413.

48. Pour la datation, voir la discussion dans Emil Schürer, The History of the Jewish People in the Age of Jesus Christ (175 B.C.-A.D. 135). Volume III, Part I : A New English Version Revised and Edited, éd. Géza Vermes, Fergus Millar et Martin Goodman, Edinburgh, T \& T Clark, 1986, p. 297-300.

49. Voir Michael Stone, Fourth Ezra: A Commentary on the Book of Fourth Ezra, Minneapolis, Fortress Press, 1990, p. 159-161. Gerson Cohen estime pour sa part que « Jacob » et «Ésaü » correspondent à des cycles historiques généraux et à des symboles archétypaux plutôt qu'à des entités historiquement définies («Esau as Symbol», p. 21). Mais il est beaucoup plus probable que le $I V^{e}$ Livre d'Esdras fasse référence à la situation vécue par les Juifs de son époque, en lien avec la destruction du Second Temple. En IV Esdras 12, 11, le quatrième royaume de Daniel 7, 23 est identifié à un aigle qui monte de la mer, et cet aigle désigne clairement Rome (cf. Stone, Fourth Ezra, p. 161, note 127). 
sa force. 19 Celui qui sort de Jacob régnera, il fera disparaître de la ville ( 'ir) (tout) survivant.

Voici comment Josèphe rapporte ce dernier oracle de Balaam (qui précède immédiatement son renvoi par le roi de Moab) :

$\mathrm{Au}$ contraire [i.e., contrairement à la demande du roi de Moab], étant tombé sur la face, il prédit les malheurs qu'éprouveraient les rois et les villes les plus célèbres (dont quelques-unes n'avaient pas encore commencé à être habitées) et tout ce qui devait arriver aux hommes par la suite, sur terre et sur mer, jusqu'à l'époque de mes propres souvenirs. En outre, puisque tout s'est réalisé comme il l'annonçait, on pourrait supposer qu'il en sera de même à l'avenir $(A . J . \mathrm{IV}, 125)^{50}$.

Josèphe ne rapporte pas le contenu de l'oracle. Mais il s'adresse à ceux qui connaissent le passage biblique, c'est-à-dire à ses coreligionnaires juifs, en glissant une allusion à la portée historique et surtout future de la prophétie des Nombres. Voici comment cette allusion peut être comprise : de même que le royaume d'Édom a été conquis par Israël au cours de l'histoire (ne serait-ce que lors de l'épisode, encore récent à l'échelle de l'histoire d'Israël, de la conquête de l'Idumée par Jean Hyrcan), dans l'avenir les ennemis d'Israël (c'est-à-dire Rome, nouvel Édom) seront à nouveau détruits. L'allusion à la «ville » ('ir en hébreu) à la toute fin de l'oracle (verset 19) a pu être interprétée en référence à l'Urbs, comme ce sera le cas par la suite dans les Targums ${ }^{51}$.

Ces allusions dans le $I V^{e}$ Livre d'Esdras et l'œuvre de Josèphe sont d'autant plus intéressantes que l'un et l'autre ont été rapprochés des milieux pharisiens ou proto-rabbiniques ${ }^{52}$. Or c'est dans la

50. Traduction d'Étienne Nodet, Flavius Josèphe. Les Antiquités juives, livres IV et $V$, Paris, Cerf, 1995, p. 31-32.

51. Voir Valentin Nikiprowetzky, «La mort d'Éleazar fils de Jaïre et les courants apologétiques dans le De Bello Judaico de Flavius Josèphe », Hommages à André Dupont-Sommer, dir. André Caquot et Marc Philonenko, Paris, Librairie d'Amérique et d'Orient - Adrien Maisonneuve, 1971, p. 461-490 (voir p. 488). La référence, dans $B . J$. VI, 289, à l'épée apparue dans le ciel, est plus difficile à interpréter comme un indice de l'identification de Rome avec Édom, malgré la référence possible à Isaïe 34,5 . En effet, dans le passage de la Guerre, ce signe est présenté comme annonçant la défaite des Juifs plutôt que celle des Romains. Il est possible toutefois que Josèphe veuille suggérer que certains Juifs l'avaient interprété par erreur comme un signe de victoire contre Rome, en identifiant dans ce cas Édom (dans Isaïe 34, 5) à Rome.

52. Au sujet du IV Esdras, voir par exemple Daniel Boyarin, «Penitential Liturgy in 4 Ezra », Journal for the Study of Judaism 3, 1972, p. 30-34 ; Gedaliah Alon, The Jews in Their Land in the Talmudic Age, trad. Gershom Levi, Jerusalem, 
littérature rabbinique que l'identification Rome - Ésaü - Édom est formulée de manière explicite ; elle y apparaît de surcroît liée au passage des Nombres mentionné précédemment, l'oracle de Balaam contre les ennemis d'Israël. Ce texte fut interprété dans un sens messianique, l'astre issu de Jacob et le sceptre issu d'Israël désignant le messie libérateur, guerrier. Durant l'épisode de la révolte de Bar Kokhba, Rabbi Aqiva aurait appliqué la prophétie de Nombres 24, 17 au chef de la révolte, en qui il aurait vu le messie attendu (le nom Bar Kokhba est en lui-même une proclamation en ce sens, puisqu'il signifie «fils de l'étoile»; ses opposants, à l'inverse, virent en lui un Bar Kozebah, c'est-à-dire un menteur) ${ }^{53}$. Dans la mesure où, d'après Nombres 24, 18, le messie «astre sorti de Jacob» est vainqueur d' «Édom», nom redoublé d'une référence à une «ville » au verset suivant, on en déduit qu'Édom devait dans ce contexte être associé à Rome.

Se posent cependant deux problèmes méthodologiques. D'une part, l'historicité de l'attribution de propos $\mathrm{X}$ à un rabbin Y est débattue, certains historiens étant prêts à admettre la validité de cette attribution dans certains cas, et d'autres la rejetant de manière quasi-systématique comme une fiction ${ }^{54}$. On trouve des rabbins qui identifient Rome à Édom dès la deuxième génération des Tannaïm, c'est-à-dire dès le début du II ${ }^{\mathrm{e}}$ siècle de n. è., mais la fiabilité de ces attributions peut être contestée. D'autre part, il convient de distinguer la période durant laquelle vécut et enseigna un sage de la date de la rédaction finale de la composition

Magnes, 1980, vol. 1, p. 52 ; Michael Stone, Fourth Ezra, p. 38. Pour Josèphe, voir Autobigraphie 12.

53. Voir Talmud de Jérusalem, Ta 'anit 4.8 pour la tradition impliquant Aqiva, et Lamentations Rabbah II, 4 pour celle autour du «menteur ». Cependant Peter Schäfer estime que l'attribution à Aqiva de la tradition relative à la messianité de Bar Kokhba est largement postérieure aux événements ; voir Schäfer, "Bar Kokhba and the Rabbis », The Bar Kokhba War Reconsidered: New Perspectives on the Second Jewish Revolt against Rome, dir. Peter Schäfer, Tübingen, Mohr Siebeck, 2003, p. 1-22.

54. Voir Hermann L. Strack et Günter Stemberger, Introduction au Talmud et au Midrash, trad. de Maurice-Ruben Hayoun, Paris, Cerf, 1986, p. 87-92. Jacob Neusner écrit : « It is obvious why we cannot proceed to outline the sequence of ideas solely on the basis of the sequence of the sages to whom ideas are attributed. We simply cannot demonstrate that a given authority really said what a document assigns to him » (Judaism in the Matrix of Christianity, p. 70). Sur l'importance accordée à l'attribution par les sources rabbiniques elles-mêmes, voir Sacha Stern, "Attribution and Authorship in the Babylonian Talmud», Journal of Jewish Studies 45/1, 1994, p. 28-51. 
dans laquelle son enseignement est cité. Si l'on trouve des rabbins du II ${ }^{\mathrm{e}}$ siècle qui associent Rome avec Édom - Ésaü, les sources qui nous rapportent ces enseignements datent pour l'essentiel, au mieux, de la fin du IV ${ }^{\mathrm{e}}$ ou du $\mathrm{v}^{\mathrm{e}}$ siècle de n. è. (si l'on s'en tient au Talmud de Jérusalem et à Genèse Rabbah). C'est pourquoi certains spécialistes considèrent que les enseignements attribués aux sages du $\mathrm{II}^{\mathrm{e}}$ ou du $\mathrm{II}^{\mathrm{e}}$ siècle dans les œuvres du $v^{e}$ doivent être vus comme des productions du ve siècle et interprétés uniquement en lien avec la christianisation de l'empire.

Il me semble pour ma part que les attributions de propos relatifs à Rome - Édom - Ésaü à des figures de la première moitié du II ${ }^{\mathrm{e}}$ siècle de n. è. ne peuvent pas être écartées simplement parce que les œuvres dans lesquelles elles apparaissent datent, dans leur rédaction finale, du v siècle. Même si l'on ne peut pas déterminer avec certitude quelle est la part de traditions anciennes véhiculées par ces œuvres et même si ces traditions ont certainement fait l'objet de reformulations, il n'en demeure pas moins que tout ne fut pas inventé au $\mathrm{IV}^{\mathrm{e}} \mathrm{ou}$ au $V^{\mathrm{e}}$ siècle $^{55}$. De plus, l'examen des textes rabbiniques relatifs à Rome montre que ces traditions sont massivement rattachées non seulement à des rabbins, mais aussi à des événements des $\mathrm{I}^{\mathrm{er}}$, $\mathrm{II}^{\mathrm{e}}$ et $\mathrm{III}^{\mathrm{e}}$ siècles ${ }^{56}$; même si certains récits ont pu être rattachés a posteriori à telle ou telle figure, cette tendance lourde témoigne malgré tout d'un aspect bien réel de la mémoire rabbinique de Rome, fermement ancrée - même plusieurs siècles après ! - dans les événements des trois premiers siècles. Enfin, certaines traditions rabbiniques sont pleinement développées dans des ouvrages tardifs, mais s'enracinent néanmoins dans la période et la littérature tannaïtiques, où on les rencontre déjà sous forme embryonnaire. Il faut donc prendre en compte l'ensemble des témoins, en ne négligeant pas ceux qui sont antérieurs à la christianisation de l'empire.

Le premier témoin rabbinique important est un passage du Sifré sur le Deutéronome, un midrash ou commentaire tannaïtique dont la rédaction finale ne dépasse pas le $\mathrm{III}^{\mathrm{e}}$ siècle de $\mathrm{n}$. è. Au $\$ 343$, en lien avec Deutéronome 33, 2 - «Il dit : YHWH est venu du Sinaï,

55. Voir également la contribution d'Hervé Inglebert dans ce dossier, montrant que la tradition d'identification de Rome avec Ésaü était connue d'Aphraate, un auteur syriaque du début $\mathrm{du} \mathrm{IV}^{\mathrm{e}}$ siècle, ce qui présuppose que cette tradition était déjà largement répandue, non seulement en Palestine mais aussi en Babylonie.

56. Pour un examen complet des sources, voir M. Hadas-Lebel, Jérusalem contre Rome. 
Il s'est levé sur eux de Séir, Il a resplendi depuis la montagne de Parân, et Il est sorti (du milieu) des myriades de sainteté ; de sa (main) droite Il a envoyé le feu de la loi »-, le midrash affirme que la Loi (Torah) ne fut pas révélée dans une seule langue, mais dans quatre langues, qui sont mises en rapport avec les quatre lieux du verset 33, 2. Le Sinaï correspond à la langue hébraïque, Séir à la langue «romaine» (romi), Parân à l'arabe, et les myriades de sainteté à l'araméen. Or, de la même manière que Parân est associé à l'arabe car il renvoie au territoire d'Ismaël (Gn 21,21), Séir est associé au « romain » car il renvoie au territoire d'Ésaü-Édom. Le midrash identifie donc ici explicitement la langue « romaine » et la réalité à laquelle elle renvoie, Rome, avec Ésaü-Édom.

Dans la Mekhilta deRabbi Ishmael, autre midrash tannaïtique dont la rédaction finale est traditionnellement datée du $\mathrm{III}^{\mathrm{e}}$ siècle, on lit par ailleurs :

(Édom) dit (à Israël) : Vous vous enorgueillissez de ce que votre père Isaac vous a légué : «La voix est la voix de Jacob» $(\mathrm{Gn} 27,22)$, «Et il (Dieu) a entendu notre voix » $(\mathrm{Nb} 20,16)^{57}$. Et nous (les Édomites), nous nous enorgueillissons de ce que notre père Isaac nous a légué : «Les mains sont les mains d'Ésaü » (Gn 27, 22), «Et tu vivras de ton épée » (Gn 27, 40). Ainsi qu'il est écrit : «Édom lui dit: Tu ne passeras pas chez moi, sinon je sortirai à ta rencontre avec l'épée » (Nb 20,18). Car ils mettent leur confiance seulement dans l'épée ${ }^{58}$.

À la différence du précédent, ce texte ne contient aucune référence explicite à Rome, mais la phrase « ils mettent leur confiance seulement dans l'épée », dans le contexte du II $^{\mathrm{e}}$ ou du $\mathrm{III}^{\mathrm{e}}$ siècle de n. è. (date de la formation du midrash), renvoie très probablement aux Romains, qui sont dans ce cas assimilés aux Édomites, descendants d'Ésaü. Il serait difficile de considérer qu'il s'agit des nations en général, car celles-ci ne sont jamais assimilées en bloc aux descendants d'Ésaü.

Cette lecture de la Mekhilta est corroborée par la tradition ultérieure d'interprétation de ce passage biblique. Dans Genèse Rabbah 65.21, les mains d'Ésaü sont associées aux crimes commis par Rome contre Israël : "Rabbi Yehudah bar Il'ai : Mon maitre interprétait: "Cette voix, c'est la voix de Jacob" (Gn 27, 22), il se lamente après ce que "les mains" lui ont fait, "les mains d'Ésaü" ». Et le texte poursuit ainsi :

57. Dans certains manuscrits on lit « la voix d'Israël » (éd. Horovitz-Rabin, p. 93).

58. MRI, Vayehi Beshalah 2 (éd. Horovitz-Rabin, p. 93). Cf. A. Schremer, Brothers Estranged, p. 125-126. 
«Rabbi Yoḥanan : La voix d'Hadrien - que (ses) os soient brisés ! exécutant à Betar 80000 myriades d'hommes $»^{59}$. Dans le contexte de ce passage, indépendamment de l'identification (possible mais non certaine) du maitre de Rabbi Yehudah bar Il'ai avec Rabbi Aqiva, il est clair que « les mains d'Ésaü » renvoient aux agissements des Romains et à la répression des révoltes juives contre Rome, en particulier celle de 132-135 (Betar est le nom de la forteresse où, d'après la tradition juive, Bar Kokhba aurait été assiégé et vaincu par les Romains).

L'interprétation de Gn 27, 22 en lien avec Rome figure également dans le passage parallèle du Talmud de Jérusalem, Ta'anit 4.8 (68d), qui rapporte plusieurs traditions associées aux événements de 132-135. Dans le ms Leiden, vient tout d'abord l'enseignement mentionné cidessus de Rabbi Yehudah bar Il'ai au nom de son maître (lequel est appelé «Barukh», probablement par erreur $\left.{ }^{60}\right)$. La version du Talmud de Jérusalem est plus claire que celle de Genèse Rabbah: «La voix de Jacob se lamente à propos de ce que lui ont fait les mains d'Ésaü à Betar » ${ }^{61}$. L'identification d' «Ésaü» avec les forces romaines ne fait ici aucun doute. Ensuite vient le passage attribuant à Rabbi Aqiva l'opinion selon laquelle Bar Kokhba était le messie, en lien avec Nombres 24, 17. Peter Schäfer estime que cette opinion remonte à la période de la révolte elle-même, mais qu'elle était à l'origine anonyme, et qu'elle ne fut attribuée à Rabbi Aqiva qu'ultérieurement ${ }^{62}$. Mais cela n'a que peu d'incidence sur l'interprétation qui identifie «Édom» en Nombres 24, 18 à Rome, puisque dans tous les cas le lien avec la révolte de Bar Kokhba est explicite. Le passage talmudique poursuit en

59. Éd. Theodor-Albeck, p. 740.

60. Cf. F. Avemarie, «Esaus Hände, Jakobs Stimme », p. 181.

61. Dans la synopse de Peter Schäfer et Hans-Jürgen Becker, ce passage correspond au \$27 (Synopse zum Talmud Yerushalmi. Band II/5-12, Ordnung Mo'ed: Sheqalim, Sukka, Rosh ha-Shana, Beșa, Ta'anit, Megilla, Hagiga und Mo'ed Qatan, Tübingen, Mohr Siebeck, 2001, p. 261). Dans le ms Darmstadt 413 Rd., le propos est attribué à Shimon bar Yohai qui le rapporte au nom de Rabbi Aqiva, et il est suivi de la phrase : "Que dit (l'Écriture) ? "Souviens-toi, YHWH, des fils d'Édom qui, au jour de Jérusalem, (disaient : Rasez, rasez jusqu'à ses fondations !)" (Ps 137, 7)».

62. Le fait que la phrase suivante, «Lorsque Rabbi Aqiva vit Bar Kozva, il s'exclama: "Celui-ci est le roi messie" », soit en araméen, est interprété par Schäfer comme le signe qu'il s' agit d'un ajout tardif, même s'il reconnaît que cela pourrait aussi être interprété comme un signe d'ancienneté. Voir Peter Schäfer, "Bar Kokhba and the Rabbis », p. $2-5$; voir aussi id., "Rabbi Aqiva and Bar Kokhba », Approaches to Ancient Judaism, dir. William Scott Green, Atlanta, Scholars Press, 1980, p. 113-130. 
rappelant et en développant l'enseignement attribué à Rabbi Yoḥanan dans Genèse Rabbah sur les 80000 myriades d'hommes tués par Hadrien à Betar. Et la suite du traité contient encore d'autres traditions relatives à la guerre de 132-135.

La destruction de 80000 myriades d'hommes à Betar est à nouveau évoquée dans le Talmud de Babylone, Gittin 57a. Surtout, une baraïta anonyme en Gittin 57b propose une interprétation de la « voix de Jacob » et des « mains d'Ésaü » $(\mathrm{Gn} 27,22)$ en rapport avec Titus (qui aurait anéanti des myriades à Alexandrie), avec Vespasien (qui serait responsable - bel anachronisme ! - de la prise de Betar), et enfin avec le « royaume impie », c'est-à-dire Rome : " "Les mains sont les mains d'Ésaü" : c'est le royaume impie, qui a détruit notre temple et brûlé notre sanctuaire, et qui nous a exilés de notre terre ». Le lien Rome -Esaü est explicite, et le fait qu'il s'agisse d'une tradition anonyme en hébreu constitue a priori un signe d'ancienneté.

Ces interprétations rapportées dans des ouvrages postérieurs à la Mekhilta deRabbi Ishmaël ne prouvent pas que tel était déjà le sens du passage dans la Mekhilta, mais la reprise et l'approfondissement du motif dans les différents textes sont néanmoins éclairants. Lorsque l'on met bout à bout tous les éléments énumérés en faveur de l'identification de Rome avec Ésaü - Édom dès la deuxième génération des Tannaïm, le résultat est significatif. Même si l'on ne peut aboutir à une certitude absolue, la probabilité que cette identification remonte à la fin du $\mathrm{I}^{\mathrm{er}}$ siècle ou à la première moitié du II $\mathrm{I}^{\mathrm{e}}$ siècle est très élevée.

On a vu que dans plusieurs sources rabbiniques, cette identification est associée à la révolte de 132-135. Il est possible que la refondation de Jérusalem comme colonie romaine (Aelia Capitolina) ait contribué à l'adoption par les rabbins de la vision audacieuse selon laquelle Rome pouvait être identifiée avec le frère jumeau d'Israël. On se trouvait en effet face à un problème qui ne se limitait pas à celui de la domination politique et militaire de Rome, mais prenait la forme d'une véritable substitution. Bien avant que le christianisme ne devienne un défi majeur, Rome posa aux Juifs le défi d'une puissance qui prend la place d'Israël, via la substitution d'Aelia Capitolina à Jérusalem - Sion. Or tel est bien le cœur du problème entre les jumeaux de la Genèse : ils rivalisent pour le droit d'aînesse et la bénédiction paternelle et divine ; il s'agit donc bien d'un problème de place et de primauté.

De manière plus générale, l'idée d'une rivalité entre Rome et Israël est pertinente bien avant que l'empire ne devienne chrétien. 
L'idéologie impériale romaine, en particulier telle qu'elle fut formulée sous Auguste par Virgile et d'autres auteurs, insistait sur la vocation singulière de Rome, choisie par les dieux depuis la nuit des temps pour dominer le monde à jamais et lui apporter la paix. Mutatis mutandis, cette vision de la destinée de l'Urbs et, par conséquent, du peuple romain, n'était pas sans rappeler certains aspects de l'élection d'Israël, en particulier dans sa dimension messianique (qui combine les éléments de la paix et de la domination universelle sur les nations). Cet aspect de la relation entre Israël et Rome n'avait d'ailleurs pas échappé à Gerson Cohen, qui écrivait (en désignant par «Rome» la Rome "païenne") :

[...] there was a basic similarity between Rome and Judea in patterns of thought and expression. Neither of them could accept their existence as a mere fact. Each considered itself divinely chosen and destined for a unique history. Each was obsessed with its glorious antiquity. Each was convinced that heaven had selected it to rule the world. Neither could accept with equanimity any challenge to its claims. [...] Given two such mutually exclusive self-appraisals, and given the confrontation on the fields of battle, it was simple to make of Rome the archfoe of all times, far worse than Nebuchadnezzar. What more appropriate picture could come to mind than Jacob and Esau contending for the same blessing ${ }^{\text {?3 }}$

L'identification de Rome avec Ésaü, le frère jumeau d'Israël, rendait intelligibles, d'un point de vue juif, la volonté de Rome de se substituer à Israël et la rivalité entre les deux peuples ; elle permettait d'expliquer le triomphe temporel de Rome, qui pouvait être perçu comme la récompense provisoire des rares actes méritants accomplis par Ésaï ; elle permettait, enfin, de réconforter les Juifs après les catastrophes de la fin du $\mathrm{I}^{\mathrm{er}}$ siècle et du début du $\mathrm{II}^{\mathrm{e}}$, en leur montrant que le conflit entre Israël et Rome avait sa cohérence propre, inscrite dans les origines mêmes d'Israël, et en les assurant de la victoire ultime contre Rome, enracinée dans la bénédiction d'Isaac à Jacob $(\mathrm{Gn} 27,29)$ et par la suite dans les oracles de jugement contre Édom.

Sil'identification de Rome avec Ésaü- Édom fait donc pleinement sens dans le contexte du $\mathrm{II}^{\mathrm{e}}$ siècle de $\mathrm{n}$. è., indépendamment du christianisme, la question demeure cependant de savoir pourquoi elle n'est pas plus fréquente et plus explicite dans les sources des trois premiers siècles. Du côté de la littérature rabbinique, on peut

63. G. Cohen, «Esau as Symbol», p. 25-26. 
évidemment avancer que les premiers midrashim, la Mishnah et la Tosefta sont pour l'essentiel des ouvrages de halakhah qui se préoccupent avant tout de codifier la vie juive et d'ériger un contremodèle positif, une alternative à l'empire ${ }^{64}$. Ils ne commentent ni les parties narratives de la Bible comme la Genèse ni les prophètes, qui contiennent la plupart des passages relatifs à Ésaü et à Édom.

Mais cet argument ne constitue sans doute pas la totalité de la réponse. Il faut rapprocher l'absence relative de textes documentant l'identification de Rome avec Ésaü - Édom d'un constat similaire formulé par Günter Stemberger à propos du récit de la destruction du Temple ${ }^{65}$. Stemberger montre que la réflexion théologique autour de cet événement dramatique ne s'est développée que progressivement, et qu'elle est beaucoup plus riche et explicite dans les ouvrages rabbiniques tardifs que dans ceux qui sont plus anciens et donc plus proches de l'événement. Il ne m'est pas possible d'analyser plus avant ce phénomène dans le cadre de cet article, mais ce constat doit nous amener à réfléchir sur l'importance de la longue durée dans la mise par écrit de la réflexion des sages sur les événements de la fin du $\mathrm{I}^{\mathrm{er}}$ siècle et du début du $\mathrm{II}^{\mathrm{e}}$.

\section{Conclusion}

En conclusion, l'identification rabbinique de Rome avec Ésaü-Édom diffère profondément du modèle des parentés bibliques ou des syngeneiai hellénistiques, qui postulaient un véritable lien généalogique, via les patriarches, entre les Juifs et d'autres peuples, mais n'identifiaient pas ces peuples avec les figures patriarcales elles-mêmes. Il faut attendre le Moyen-Âge et le Sefer Yosippon pour que la parenté entre Israël et Rome - Édom - Ésaü soit pensée sur le modèle des parentés hellénistiques, et les Romains présentés comme de véritables descendants des Édomites. Plutôt qu'une généalogie commune, l'identification de Rome avec Ésaü - Édom met en avant une typologie.

64. Cf. F. Avemarie, «Esaus Hände, Jakobs Stimme», p. 184. Sur la Mishnah comme alternative à l'empire, voir Ishay Rosen-Zvi, "Rabbis as Romans Revisited », Jewish Cultural Encounters in the Ancient Mediterranean and Near Eastern World, dir. Mladen Popovic, Groningen, 2015 (à paraître).

65. Voir Günter Stemberger, «Reaktionen auf die Tempelzerstörung in der rabbinischen Literatur», Zerstörungen des Jerusalemer Tempels: GeschehenWahrnehmung-Bewältigung, dir. Johannes Hahn, Tübingen, Mohr Siebeck (WUNT 147), 2002, p. 207-236. 
Le choix d'identifier Rome avec le frère jumeau d'Israël était pour le moins paradoxal et lourd d'implications, puisque, pris au pied de la lettre, il impliquait une proximité et un lien intime entre Rome et Israël. Cela correspondait nécessairement à un choix réfléchi de la part des rabbins. Ceux-ci avaient peut-être hérité d'une tradition préexistante, dont on perçoit des échos hésitants dans le IV ${ }^{e}$ Livre d'Esdras et l'œuvre de Flavius Josèphe, mais c'est dans la littérature rabbinique que cette identification devint explicite et, avec le temps, massive. À la suite de Gerson Cohen, il me semble que la typologie Rome - Ésaü - Édom reflète la perception qu'eurent certains rabbins de la proximité et de la rivalité entre Israël et Rome. En effet, seule la conscience d'une rivalité dans la proximité pouvait rendre cohérent le choix de la figure d'Ésaü pour désigner Rome. Comme dans le cas des généalogies bibliques et des syngeneiai hellénistiques, la typologie Rome - Ésaü - Édom permit aux rabbins d'attribuer à Rome une place symbolique dans une représentation juive du monde et de compenser la faiblesse politique d'Israël par une prise de pouvoir symbolique consistant à assigner à l'autre une place et, simultanément, une destinée. Mais elle permettait aussi de faire sens de cette étrange et paradoxale ressemblance entre les vocations proclamées d'Israël et de Rome.

Carol Bakhos insiste à juste titre sur le fait que la référence à Ésaü dans la littérature rabbinique revêt des fonctions diverses, et qu'il peut représenter un "ultimate Other » sans arrière-plan historique particulier. Toutes les occurrences d' «Ésaü » ou «Édom» ne sont pas à interpréter en lien avec Rome ${ }^{66}$. Elles sont toutefois nombreuses à pointer dans cette direction, et peut-être même plus précisément dans la direction de la Rome païenne des trois premiers siècles, plutôt que dans celle de la Rome chrétienne. L'examen des textes qui évoquent des événements liés à Rome ou des realia romaines indique en effet que la mémoire rabbinique de Rome est étroitement liée aux événements des trois premiers siècles. Cela étant, la christianisation de l'empire conféra certainement un relief nouveau à l'identification de Rome avec Ésaü - Édom et à sa rivalité avec Israël.

berthelot@mmsh.univ-aix.fr

66. Carol Bakhos, «Figuring (out) Esau: The Rabbis and Their Others », Journal of Jewish Studies 58/2, 2007, p. 250-262. 